\title{
Optimal Power Management for Energy Harvesting Systems with A Backup Power Source
}

\section{Conference Paper}

\section{Author(s):}

Draskovic, Stefan; Thiele, Lothar

Publication date:

2021

Permanent link:

https://doi.org/10.3929/ethz-b-000496594

Rights / license:

In Copyright - Non-Commercial Use Permitted

Originally published in:

https://doi.org/10.1109/meco52532.2021.9460139 


\title{
Optimal Power Management for Energy Harvesting Systems with a Backup Power Source
}

\author{
Stefan Draskovic, Lothar Thiele \\ Computer Engineering and Networks Laboratory (TIK), ETH Zürich, Switzerland \\ Email: stefandr@ethz.ch, thiele@ethz.ch
}

\begin{abstract}
Energy harvesting has been extensively used to allow for long-term and unattended operation of nodes in large-scale distributed systems. However, the smaller the relative rechargeable energy storage of the harvesting system, the higher is the sensitivity of its operation to short-term non-deterministic changes of the harvested power or the current power demand. A reliable and predictable node operation can be achieved with an additional backup battery. First commercial products that allow for an efficient energy exchange between energy harvester, backup battery and energy consumer are available. But, there are no energy management algorithms that optimize the longterm node utility while maximizing the lifetime of the node in terms of its backup battery. We formally model the problem, provide an optimal solution for a class of node utility functions, and implement the strategy on a resource-constrained hardware. Extensive simulations as well as experiments on indoor solar data traces show the accuracy of the abstract model, the run-time overhead and efficiency of our approach.
\end{abstract}

\section{INTRODUCTION}

Energy harvesting is a key technology to provide longterm, autonomous and sustainable energy to embedded devices in large-scale distributed systems. A large body of results shows the potential of extracting energy from light, temperature differences, acceleration, vibration, and electromagnetic waves, see for example [1]. Motivated by cost, size, safety and environmental reasons, there is a recent trend to substantially reduce the necessary local rechargeable energy storage, often in the context of transient and intermittent computing [2]. This approach also allows to replace rechargeable batteries with super-capacitors that are accompanied by advantages such as increased safety, a high number of charge-discharge cycles and high retrieval efficiency.

The smaller the relative maximal energy storage capacity of the node, the higher is the sensitivity of its operation to shortterm non-deterministic changes of the harvested power or the power demand. Concepts to decrease the sensitivity and to deal with frequently interrupted processing have been developed [3], [4], including extreme approaches where the harvesting source is directly coupled to the node [5].

Nevertheless, there are many applications where correct operation requires the unconditional availability of some amount of energy within a given time interval. Examples are automatic control, surveillance, early warning, or safety-critical sensing applications. In addition, low-power multi-hop networks typically need periodic refresh operations for synchronization. Unless there is a reliable correlation between harvested energy on the one hand and events to be sensed or actions to be performed on the other, the usefulness of intermittent or transient approaches to energy provisioning in critical applications is limited.

A reliable and predictable node operation can be obtained with an additional backup battery, a primary cell for example. This solution not only has been suggested by academia, e.g., [6]-[8], but industry is also providing first commercial products that allow for an efficient energy exchange between energy harvester, backup battery and energy consumer [9]. Such a design not only allows an otherwise unreliable system to operate without interruption, but also offers additional benefits such as increasing the efficiency and speed of cold-start from an empty energy storage, retaining state across restarts, or operating without interruption for years or decades.

One of the most important aspects in energy harvesting nodes is the energy management system. It is responsible for deciding on the energy flow between the energy source, the rechargeable energy storage unit and the energy consumer. It also controls the configuration and operation of the consumer such that an overall system utility is maximized. Examples of varying operation of the consumer are adapting the sensing rate, the communication rate, the kind of data processing algorithms used or even going to a low energy "energy scarcity" mode. Surprisingly, energy management and control has been extensively covered in the general sense of energy harvesting sensing systems, see [10]-[14], but no results are available in the context of an additional backup battery.

The present paper fills this gap by providing models and methods that provide answers to the following question: How to control the operation of a harvesting node with a backup battery such that a minimal amount of energy is guaranteed, its lifetime is maximized and its long-term utility is optimized? As a result, the described concepts and hardware for energy harvesting nodes that include a backup battery can now be combined with control algorithms to make optimal use of the available resources such as the backup battery, the harvested energy and the energy storage capacity. In summary, the paper contains the following new results:

- A formal model describing a complete harvesting system.

- An algorithm that optimizes the long-term node utility while guaranteeing a minimal available energy and maximizing the life-time of the system, and an extension to this algorithm that copes with non-perfect estimations of future energy based on model predictive control.

- An implementation of the overall hardware and software, a characterization of its essential properties and an approach to include them in the abstract model. Extensive emulation 
and experimental results on indoor solar data that show the applicability of the approach, as well as the accuracy of the abstract model.

\section{RELATED WORK}

Energy Systems. Superficially, there is a close relation to energy systems incorporating renewable sources like solar and wind. Whereas one can draw analogies in terms of energy generation, energy storage (water tanks, car batteries), application control (demand side management), there are essential differences in terms of models and methods due to the incorporation of the energy grid, sufficient energy for implementing complex control strategies, energy conversion and transmission, consumer behavior and cost functions, see [15], [16].

Existing Hardware Implementations. A plethora of nodes featuring photovoltaic energy harvesting as well as backup batteries exist. For example, [7] presents a WSN (wireless sensor network) solution for tracking of goods in a commercial seaport. In [6], a general purpose WSN has a large lithium ion rechargeable battery, while a primary battery is used to ensure uninterrupted operation. A WSN optimized for harvesting energy in low illumination environments [17] features a similar approach. Hardware components for energy management systems with backup batteries exist, from the bq25505 energy management chip [9] featuring a simple multiplex, to the smart platform [8] enabling complex energy management functions. Still, there are no results available that allow to control the operation of the node and the energy management system such that the backup battery is used as little as possible while maximizing the long-term utility of the node.

Energy-Neutral Operation. A first work on dynamic power management was written by Kansal et al. [10], where an analytical model featuring ENO (energy-neutral operation) constraints was developed with the goal of maximizing performance. The problem of computing the dynamic power management profile efficiently was explored in [14]. When ample historical data about the harvestable energy is available, a power management scheme proposed in [13] gives probabilistic guarantees that a minimal energy utilization will always be realized. Examples of uninterrupted environmental monitoring nodes operating over multiple years are given in [18], [19] for a high-mountain environment, in [20] for a remote field, and in [21] for a deep forest scenario. Even though these works feature detailed design principles and power management procedures, uninterrupted operation is not guaranteed under a non-deterministic harvesting environment. In addition, none of these results considers the availability of a backup battery.

Transient Systems. Transiently powered computing systems are designed with little or no energy buffers, thus working only when sufficient energy is available, while retaining their state otherwise [22]. Generic energy management units for these systems exist [3]. Examples of use-cases include a passive camera powered by RFID [23], or an AC power meter powered by the current it is measuring [24]. Due to its low overhead, state retention is often realized in FRAM technology [5], [25]. Furthermore, transient systems feature complex intermittent programming models, i.e., [4], [26]. However, these systems

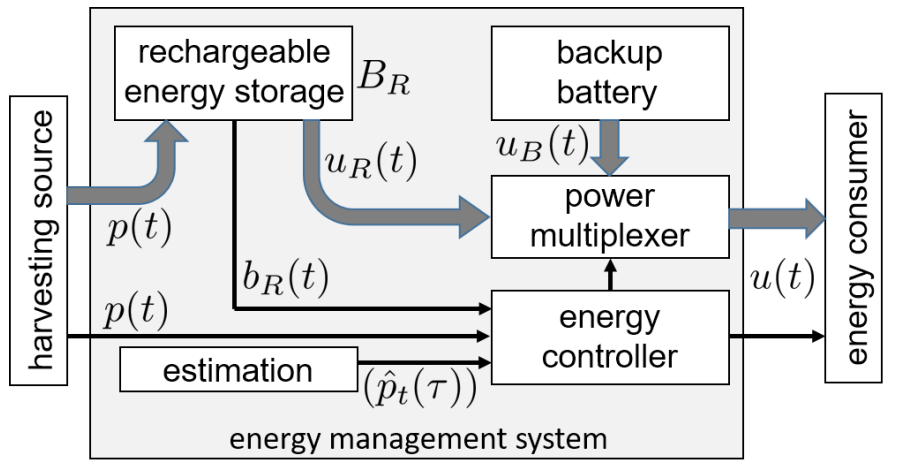

Fig. 1. Overview of the abstract model of the energy management system with a backup battery. The thick and thin arrows show the energy and information flow, respectively.

may remain offline for extended periods of time, which is only acceptable for a reduced set of application scenarios.

\section{SYSTEM MODEL}

1) Basic Assumptions: We start with the introduction of an abstract model which is the basis for energy control. In later sections, the model will be refined in order to take into account non-ideal behavior of the hardware-software system.

An abstract overview of the overall setup is shown in Figure 1. We use a discrete time system model, where each time instance $t$ is an integer $t \in \mathbb{Z}_{\geq 0}$. The energy management system has two types of energy reservoirs: a rechargeable energy storage and a backup battery. The rechargeable energy storage is replenished by an energy harvester, while the backup battery is a primary battery. The rechargeable energy storage has $b_{R}(t)$ energy stored at time $t$, and a maximum capacity $B_{R}$. For our initial abstract model, we suppose it is loss-free, extensions are described in sec. VI.

The harvested energy during time interval $[t, t+1)$ is denoted as $p(t)$. This value depends on the energy harvester and the environment. The consumed energy during time interval $[t, t+1)$ is denoted as $u(t)$, with $u$ being the use function. The energy management system provides the energy consumer with information about the target energy consumption for the next time interval. As a consequence, the consumer adapts its operation to match this target value, for example by changing its sensing rate, the processing speed, or the like. The consumed energy $u(t)$ equals the energy taken from the rechargeable energy storage and from the backup battery, namely $u(t)=$ $u_{R}(t)+u_{B}(t)$. The energy management system also decides which portion is taken from which energy reservoir. The base consumption $u_{\mathrm{BASE}} \geq 0$ defines a lower bound on the consumed energy with $u(t) \geq u_{\mathrm{BASE}}, \forall t \in \mathbb{Z}_{\geq 0}$.

For the rechargeable energy storage, the following holds: $b_{R}(t+1)=\min \left\{b_{R}(t)+p(t)-u_{R}(t), B_{R}\right\}$. The harvested energy that is discarded because the rechargeable energy storage is full is called "wasted energy" for short. The use of the backup battery in time interval $\left[t_{1}, t_{2}\right)$ is denoted as $E\left(t_{1}, t_{2}\right)=\sum_{t_{1} \leq t<t_{2}} u_{B}(t)$.

A utility function is strictly concave function $\mu: \mathbb{R}_{\geq 0} \rightarrow$ $\mathbb{R}_{>0}$. It models the diminishing return of investment if the 
energy consumption of a node grows. The total utility in a time interval $\left[t_{1}, t_{2}\right)$ is the sum of the utility during this time, i.e., $U\left(t_{1}, t_{2}\right)=\sum_{t_{1} \leq \tau<t_{2}} \mu(u(\tau))$.

2) Initial Problem Specification: We solve the optimization problem in two steps. At first, we assume that we know the harvested energy in the future. This forms the basis for the model predictive energy control, which is the case when the future harvested energy is only estimated.

Given Quantities. We are given the minimal required energy consumption $u_{\mathrm{BASE}}$, the initial and the final rechargeable energy storage states $b_{R}(0)$ and $b_{R}(T)$, and the harvested energy $p(t)$ for all $t \in[0, T)$.

Constraints. The following constraints hold for the (abstract) energy management system for all $t \in[0, T)$ :

$$
\begin{gathered}
u_{B}(t) \geq 0 \quad ; \quad u_{R}(t) \geq 0 \\
u(t)=u_{R}(t)+u_{B}(t) \geq u_{\mathrm{BASE}} \\
0 \leq b_{R}(t+1)=b_{R}(t)+p(t)-u_{R}(t) \leq B_{R}
\end{gathered}
$$

Optimality. Under the above constraints, an optimal energy controller determines a rechargeable use function $u_{R}^{*}(t)$ and backup use function $u_{B}^{*}(t)$ that satisfy the following criteria:

01: The total use of the backup energy $E^{*}(0, T)$ is the smallest among all possible use functions, and therefore, the lifetime of the node is maximized.

02: Among the use functions that satisfy the above criterion, the use function $u^{*}(t)=u_{R}^{*}(t)+u_{B}^{*}(t)$ has the maximal total utility $U^{*}(0, T)$.

The optimal solution to the clairvoyant problem is given in sec. IV. Based on finite horizon control, a solution to the problem with unknown future energy is given in sec. V.

\section{Optimal Clairvoyant Solution}

As mentioned before, results are available that construct an optimal energy control algorithm but without considering the presence of a backup battery nor the goal of maximizing the lifetime of the node. In this section, we first restate a result shown in [27]. It provides a first step towards understanding the optimal use of a backup battery. Subsequently, we present a unique optimal use function $u^{*}(t)$ which is the solution to the complete optimization problem (1), meaning it has the minimal total backup energy usage (O1) and among all functions fulfilling these conditions, it has the largest possible utility $(\mathrm{O} 2)$.

\section{A. Properties of the Optimal Solution}

We start with the simple case without a backup battery, and with no required base consumption, i.e., $u_{\mathrm{BASE}}=0$. The corresponding simplified problem is

$$
\begin{gathered}
u_{R}(t) \geq 0 \\
0 \leq b_{R}(t+1)=b_{R}(t)+p(t)-u_{R}(t) \leq B_{R}
\end{gathered}
$$

for all $t \in[0, T)$, with given $b_{R}(0), b_{R}(T)$ and $p(t)$. The objective is to maximize the minimal energy used from the rechargeable storage $u_{R}(t)$, and among those use functions to choose the one which maximizes the utility $U_{R}(0, T)=$ $\sum_{0 \leq t<T} \mu\left(u_{R}(t)\right)$. The following has been shown in [27]:
Theorem 4.1: Given a use function $u_{R}^{*}(t)$ that satisfies the conditions in (2). If the relations

$$
\begin{gathered}
u_{R}^{*}(t-1)<u_{R}^{*}(t) \Longrightarrow b_{R}^{*}(t)=0 \\
u_{R}^{*}(t-1)>u_{R}^{*}(t) \Longrightarrow b_{R}^{*}(t)=B_{R}
\end{gathered}
$$

hold for all $t \in[1, T)$, then $u_{R}^{*}(t)$ maximizes the minimal used energy, it maximizes the total utility $U_{R}(0, T)$, and it is unique.

With this knowledge, we now demonstrate how to take an optimal solution $u_{R}^{*}(t)$ to the partial optimization problem in (2), and obtain from it an optimal solution $u^{*}(t)$ to the complete optimization problem in (1). We show in the following, that an optimal solution $u_{R}^{*}(t)$ to the simplified problem as defined in (2) leads to an optimal solution of the clairvoyant problem with a backup battery and minimal use function $u_{\text {BASE }}$ with

$$
\begin{gathered}
u^{*}(t)=u_{R}^{*}(t)+u_{B}^{*}(t) \\
u_{B}^{*}(t)=\max \left\{0, u_{\mathrm{BASE}}-u_{R}^{*}(t)\right\}
\end{gathered}
$$

for all $t \in[0, T)$.

This fundamental result is shown with the following two Lemmas. The corresponding proofs are contained in the technical report. The first Lemma shows that the solution in (3) minimizes the use of the backup battery and therefore, maximizes the lifetime of the node.

Lemma 4.2: Given an optimal solution $u_{R}^{*}(t)$ to the simplified optimization problem in (2). Then $u^{*}(t)=u_{R}^{*}(t)+u_{B}^{*}(t)$ with $u_{B}^{*}(t)=\max \left\{0, u_{\mathrm{BASE}}-u_{R}^{*}(t)\right\}$ for all $t \in[0, T)$ has the minimal total backup usage $E^{*}(0, T)$ among all solutions to the optimization problem (1) with base consumption $u_{\mathrm{BASE}}$.

The next Lemma shows that the relations in (3) maximize the total utility, i.e., $U^{*}(0, T)$ is maximal for all use functions $u^{*}(t)$.

Lemma 4.3: Given an optimal solution $u_{R}^{*}(t)$ to the simplified optimization problem in (2). Then $u^{*}(t)=u_{R}^{*}(t)+u_{B}^{*}(t)$ with $u_{B}^{*}(t)=\max \left\{0, u_{\mathrm{BASE}}-u_{R}^{*}(t)\right\}$ for all $t \in[0, T)$ has the maximum total utility $U^{*}(0, T)$ among all solutions to the optimization problem (1) with base consumption $u_{\text {BASE }}$.

We now proceed to demonstrate possibilities to efficiently solve the energy management problem on resource-restricted devices, as the solution to this clairvoyant problem is a prerequisite for (a) a practical solution that does not require the knowledge of the harvested energy in the future and (b) considering implementation artifacts.

\section{B. Computing the Optimal Solution}

The solution to the problem defined in sec. III-2 still needs to be computed. Efficient algorithms for deriving the solution to the simple optimization problem (2) are known from previous work, see [27], [28]. Therefore, there is only one main problem to be solved. How can we efficiently implement (3) such that switching constraints as present in typical hardware implementations are taken into account? Following (3), the overall use function for the energy consumer is given by $u^{*}(t)=\max \left\{u_{R}^{*}(t), u_{\mathrm{BASE}}\right\}$ and it is unique, see Theorem 4.1. Nevertheless, there is some flexibility in partitioning $u^{*}(t)$ into its backup battery $u_{B}^{*}(t)$ and rechargeable energy storage $u_{R}^{*}(t)$ components. 


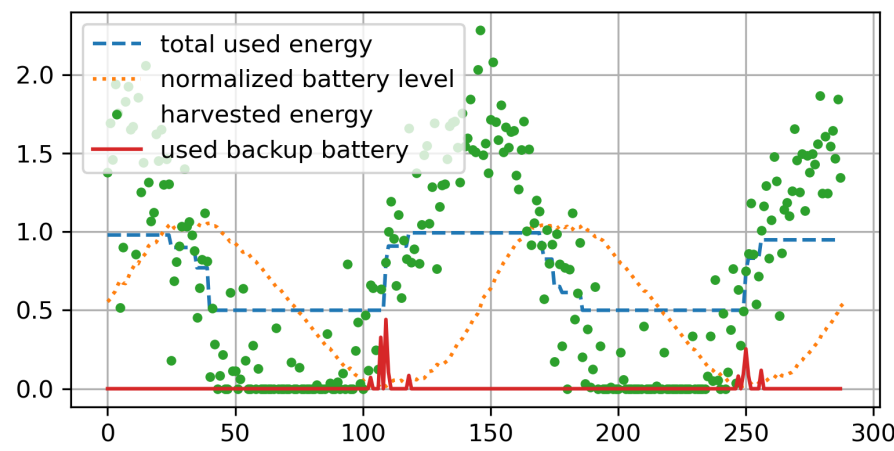

Fig. 2. The unique optimal solution $u^{*}(t)$ (total used energy) to the optimization problem, the function $u_{B}^{*}(t)$ (used backup battery) and the corresponding energy in the rechargeable energy storage (battery level normalized to $B_{R}$ ).

Let us use the term switching interval as a lower bound on the time between switching from using the backup battery to using the rechargeable energy storage and vice versa. Now, there are two questions to be answered: What is a suitable switching algorithm that determines the energy source for the next time interval? And what is a suitable switching interval? Due to space constraints, we just provide a sketch of the algorithm: We use the backup battery only if there is the danger of the rechargeable energy storage being depleted, and this is checked every switching interval. This approach is not optimal, of course, but it can be shown that optimality is regained if the capacity of the battery is increased by an easily computable amount. For realistic scenarios, this increase is practically negligible and on the order of a few percent.

Example. We end this section with an example to illustrate the aforementioned use functions and their optimization. In Figure 2, an energy harvesting function $p(t)$ is shown, while $u_{\mathrm{BASE}}=0.5$ and $B_{R}=24$. The total simulation horizon is 288 time steps. One can see how the optimal total use function $u^{*}(t)$ responds to the changes in the harvested input energy, and how it is never less than the base consumption $u_{\mathrm{BASE}}$. The diagram also visualizes the partitioning of the use function as the amount of energy drawn from the backup battery $u_{B}^{*}(t)$ is shown as well. We use the discretized selection algorithm as explained in this subsection with $S=1$, i.e., we execute it for every time step.

\section{FINITE HORIZON CONTROL}

In a realistic setting, a perfect estimate of future harvested energy is not available. Therefore, we employ the well known concept of model predictive control with a finite horizon, as used in [13], [29]. In contrast to the optimal clairvoyant solution shown before, this heuristic solution can cope with deviations between the predicted and actual harvested, or scheduled and actually used energy. We outline the concept of Finite Horizon Control here and refer the reader to the technical report for implementation details.

The concept is rather intuitive: At every time step $t$, the optimal use functions for the rechargeable energy storage and the backup battery are computed based on the current energy in the rechargeable storage and energy predictions. We only apply the optimal use functions in the time interval $[t, t+1)$.
At time $t+1$ and all future time steps, we repeat the same process. The optimal use functions are determined for a finite horizon of length $T$, namely for the time interval $[t, t+T)$. If $T$ is sufficiently large and the energy predictions are accurate, then the finite horizon control matches the optimal solution.

The finite horizon scheme demands substantial computational resources. As this is not easily available on all embedded devices, we reference here an implementation based on LookUp Tables [14] which greatly reduces the run-time computational requirements. For a given estimate, the optimal use at time $t$ for a set of energy storage levels $b_{R}(t)$ is determined. Therefore, for a fixed or periodic prediction of harvested energy, just a few parameters need to be stored to cover all initial rechargeable energy storage levels. In case of a reasonably small set of different predicted harvested energy traces, the necessary amount of storage and computation is small. For example, in the scenario explored in sec. VIII, the basic time interval is 10 minutes and the finite horizon period $T$ is one hour. If one assumes a quadratic approximation is used for non-precomputed energy storage levels, then we have 6 different predicted harvested energy traces, and for each of them just three 32 Bit values need to be stored, which results in $4 \cdot 3 \cdot 6=72$ Bytes. If one assumes 25 discrete values for the rechargeable energy storage level, instead of using an approximation, one would have to store $6 \cdot 4 \cdot 25=600$ Bytes.

\section{NON-IDEAL SySTEM Model}

Starting from our simple system model in sec. III we considered already two implementation artifacts, namely that it is only possible to draw energy from one of the two energy sources, and that the harvested and consumed energy may differ from estimations or consumption goals, respectively (finite horizon control). Here, we describe how to adapt the model in order to incorporate inefficiencies that may exist in the power management hardware without affecting the optimality of the solution.

Constraints The following non-ideal behavior is modeled, see also sec. VII-2:

- If the harvesting device generates energy $\tilde{p}(t)$ in $[t, t+1)$, energy in the rechargeable energy storage only increases by $\sigma_{p} \cdot \tilde{p}(t)$.

- If the load uses energy $\tilde{u}_{R}(t)$ in $[t, t+1)$, then the energy stored in the rechargeable energy storage decreases by $\sigma_{R} \cdot \tilde{u}_{R}(t)$. In addition, there is a leakage $\delta_{R}$ of the rechargeable energy storage.

- If the load uses backup energy $\tilde{u}_{B}(t)$ in $[t, t+1)$, then the energy stored in the backup battery reduces by $\sigma_{B} \cdot \tilde{u}_{B}(t)$. In addition, there is a leakage $\delta_{B}$ of the backup battery.

The last item is mainly relevant for computing the lifetime $L$ of the node. It can be determined using the initial energy $B_{B}$ in the backup battery and the accumulated use of the backup battery $E(0, L)=\sigma_{B} \cdot \sum_{t \in[0, L)} \tilde{u}_{B}(t)$ as follows: $B_{B}=E(0, L)+$ $L \cdot \delta_{B}$.

These extended relations consider the mentioned implementation artifacts:

$$
\tilde{u}_{R}(t) \geq 0 \quad ; \quad \tilde{u}_{B}(t) \geq 0
$$




$$
\begin{gathered}
\tilde{u}(t)=\tilde{u}_{R}(t)+\tilde{u}_{B}(t) \geq \tilde{u}_{\mathrm{BASE}} \\
0 \leq \tilde{b}_{R}(t+1)=\tilde{b}_{R}(t)+\sigma_{p} \cdot \tilde{p}(t)-\sigma_{R} \cdot \tilde{u}_{R}(t)-\delta_{R} \leq \tilde{B}_{R}
\end{gathered}
$$

If we perform the variable transformations (5), then we directly obtain from (4) the abstract relations in (1) and thus, all results which refer to the original optimization problem apply as well.

$$
\begin{gathered}
u_{R}(t)=\sigma_{B} \cdot \tilde{u}_{R}(t) ; u_{B}(t)=\sigma_{B} \cdot \tilde{u}_{B}(t) ; u(t)=\sigma_{B} \cdot \tilde{u}(t) \\
u_{\mathrm{BASE}}=\sigma_{B} \cdot \tilde{u}_{\mathrm{BASE}} ; B_{R}=\frac{\sigma_{B}}{\sigma_{R}} \tilde{B}_{R} \\
b_{R}(t)=\frac{\sigma_{B}}{\sigma_{R}} \tilde{b}_{R}(t) ; p(t)=\frac{\sigma_{B}}{\sigma_{R}}\left(\sigma_{p} \cdot \tilde{p}(t)-\delta_{R}\right)
\end{gathered}
$$

\section{IMPLEMENTATION AND CHARACTERIZATION}

Here we demonstrate the accuracy of the non-ideal model, as presented in sec. VI, by describing and characterizing an implementation of an energy harvesting embedded system with a backup battery.

1) Hardware and Software Setup: The hardware is shown in Figure 3, and consists of a photovoltaic energy harvester, a power management unit, a super-capacitor as the rechargeable energy storage, a DC power supply as the backup battery, and a microcontroller unit as the load. The photovoltaic panel is an AM-5412 from SANYO Semiconductor. A $C^{\mathrm{SC}}=5 \mathrm{~F}$ super-capacitor is used, and during operation its voltage ranges between $3.3 \mathrm{~V}$ and $4.3 \mathrm{~V}$. The backup battery was emulated with a DC power supply, providing a constant $3.3 \mathrm{~V}$ when needed. Primary batteries like the SAFT LS 14500 show an almost constant voltage throughout their lifetime. Therefore, there is no need to consider additional, time-dependent efficiency. In addition, a battery's temperature-dependent capacity impacts the system's lifetime, but does not change the optimality of our approach.

The power management unit consists of a bq25505 harvesting management chip [9], and a TPS62740 step-down converter. The bq25505 harvesting management chip performs passive maximum power point tracking for maximum energy transfer from the photovoltaic panel to the super-capacitor. The chip is also a power multiplexer, switching between the rechargeable and backup batteries in the following way. If the super-capacitor's voltage is lower than $3.3 \mathrm{~V}$, the chip switches to the backup battery. And when the super-capacitor's voltage is larger than $3.4 \mathrm{~V}$, the chip switches to the rechargeable energy storage. In practice, this means that the backup battery is used while the super-capacitor's voltage increases from $3.3 \mathrm{~V}$ to $3.4 \mathrm{~V}$. This operation follows the model described in sec. IV-B. The microcontroller unit is a MSP432P401R board from Texas Instruments, commonly found in embedded applications. Two tasks are implemented on it, the 'power manager' and the 'adaptive consumer'. The 'power manager' implements the scheduling scheme and harvested energy estimator. This task includes sampling of the super-capacitor's voltage, which allows to determine the currently stored energy $b_{R}(t)$. The sampling is done by connecting an ADC port to the super-capacitor through an appropriate high-resistance voltage divider. The 'adaptive consumer' can consume different energy values, which emulates sensing or communicating with different rates, different data processing algorithms or actuating LEDs.

2) Characterization: To characterize the described system, we conduct four experiments. Interpreting results of these experiments, we express the harvested energy as a function of illumination, and quantify the parameters of the non-ideal model as presented in sec. VI.

Experiments involving illumination are done in a laboratory environment, where a solar test-bed [30] recreates the desired illumination levels. The test-bed completely encloses a photovoltaic panel, such that it is exposed to a desired illumination trace using a programmable light source. All measurements are made using a RocketLogger [31], which monitors various voltage and current channels in parallel. Most importantly, the PV panel's harvested power $P_{\text {out }}^{\mathrm{PV}}$ and the microcontroller's power consumption $P_{\text {in }}^{\mathrm{MSP}}$ is obtained through voltage and current measurements, while the rechargeable energy storage's voltage $V^{\mathrm{SC}}$ is used to estimate the net power flow in the rechargeable energy storage $P_{\mathrm{est}}^{\mathrm{SC}}: P_{\mathrm{est}}^{\mathrm{SC}}=C^{\mathrm{SC}} / 2 \cdot \frac{\partial}{\partial t} V^{\mathrm{SC}^{2}}$. The most important details, and results of the characterization are given below, while specifics are found in the technical report. PV Panel Characterization. We first characterize the PV energy harvesting panel together with the bq25505 which performs passive maximum power point tracking. This enables us to model how much energy is harvested depending on the illumination level. A total of 13 illumination levels are used, from 0 to $120 \mathrm{kLux}$ in even steps. The following linear fit can be made: $P_{\text {out }}^{\mathrm{PV}}=0.3791 \cdot 10^{-3} \frac{\mathrm{W}}{\mathrm{lux}} \cdot L$. This fit is used in sec. VIII to create a harvested energy trace $p(t)$ from a trace of measured illumination.

Charging Characterization. Here we characterize the $\sigma_{p}$ parameter of the non-ideal model, see sec. VI, which encapsulates losses in power point tracking on the bq25505, as well as charging losses of the super-capacitor. The characterization is performed with the microcontroller and linear regulator disconnected. First, we expose the PV panel to various illumination levels, in order to measure the power produced by the PV panel $P_{\text {out }}^{\mathrm{PV}}$, as well as the power stored in the rechargeable energy storage $P_{\text {est }}^{\mathrm{SC}}$. Then we construct a linear fit that minimizes the root-mean-square error and links the harvested power $P_{\text {out }}^{\mathrm{PV}}$ and the stored power $P_{\text {est }}^{\mathrm{SC}}: P_{\mathrm{est}}^{\mathrm{SC}}=0.8529 \cdot P_{\text {out }}^{\mathrm{PV}}$. The fit is not perfect due to measurement noise as well as other factors such as a non-linear behavior of the power point tracking. Still, the fit is highly accurate with a root mean square error of only $4.313 \cdot 10^{-4}$. As a result, we can model the charging efficiency of this particular implementation as $\sigma_{p}=0.8529$.

Discharging Characterization. Now we present the characterization of the $\sigma_{R}$ parameter of the non-ideal model, see sec. VI, which quantifies the loss in power of the linear regulator, the power multiplexer, as well as the discharging losses of the super-capacitor. In this measurement setup the energy harvesting PV panel is disconnected, and the microcontroller is set to consume at various power levels. We measure the power consumed by the microcontroller $P_{i n}^{\mathrm{MSP}}$ and the power discharged from the super-capacitor $P_{\mathrm{est}}^{\mathrm{SC}}$. A linear fit that minimizes the root-mean-square error yields $P_{\text {est }}^{\mathrm{SC}}=1.0789 \cdot P_{\text {in }}^{\mathrm{MSP}}$. Again, the 


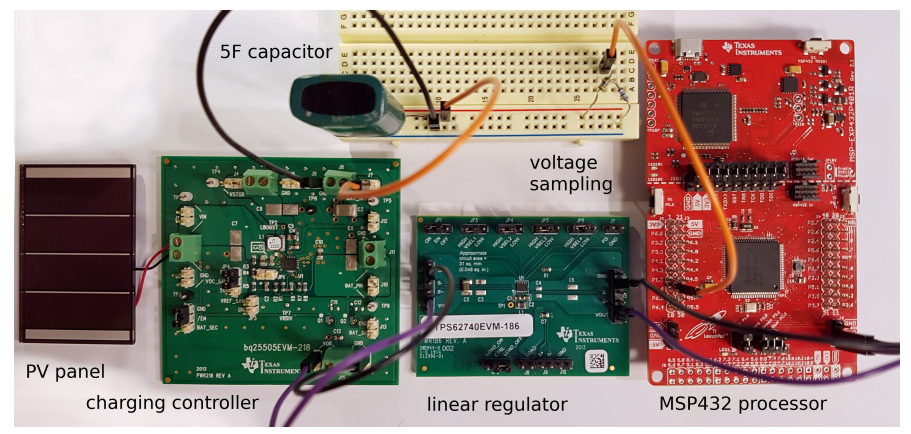

Fig. 3. Components of our hardware implementation of the energy management system with a backup battery. Backup battery not visible.

fit appears to be sufficiently accurate as the root mean square error is $1.020 \cdot 10^{-3}$. As a result, the discharging efficiency for our implementation is set to be $\sigma_{R}=1.0789=1 / 0.9269$.

Rechargeable Energy Storage Leakage Characterization. The final measurement quantifies the decrease in stored energy caused by the super-capacitor's internal leakage and the sampling of the super-capacitor's voltage. Initially, the rechargeable energy storage was fully charged, and both the PV energy harvester and the consumers were disconnected. Over 38 hours, an average power dissipation of $P_{\mathrm{est}}^{\mathrm{SC}}=93.50 \mu \mathrm{W}$ was observed. As the value is low relative to the chosen experimental setup, this leakage is not taken into account in the modeling of the system.

3) On-line Computational Overhead for Finite Horizon Energy Management: We evaluated the direct implementation of the finite horizon scheme using the clairvoyant algorithm from [28]. An implementation on the chosen MSP432 microcontroller resulted in the following findings: For $T=8 h$ horizon, using single-precision floating point arithmetic, and permitting an $\epsilon=0.01$ error in the use function $u$ which corresponds roughly to an error of $0.5 \%$ relative to the maximum value, it takes 0.2 seconds to compute $u(t)$.

\section{TRACE-BASED EXPERIMENTS}

In this section we use simulations to illustrate the difference between the optimal clairvoyant solution $u^{*}(t)$, the finite horizon solution $u^{F}(t)$, and a baseline simple heuristic approach that does not make use of energy predictions. In addition to the scenario described below, we also investigated a hypothetical system deployed in a remote outdoor area with the goal of operating for multiple years, as in many previous results such as [13], [27]. The corresponding results are described in the technical report.

The finite horizon control algorithm uses the EWMA estimator for predicting the future energy. EWMA [32] is a simple yet widely used estimation algorithm, which estimates future energy as an exponentially weighted moving average of energy harvested in previous time instances. The simple baseline control algorithm follows the purely reactive scheme ENOMAX [11], where the system tries to maintain a predefined state of charge of the rechargeable energy storage $c \cdot B_{R}$. It can be described as follows: $\left.u^{N}(t)=\max \left(u_{\mathrm{BASE}}, b_{B}(t)-0.6 \cdot B_{R}\right)\right)$

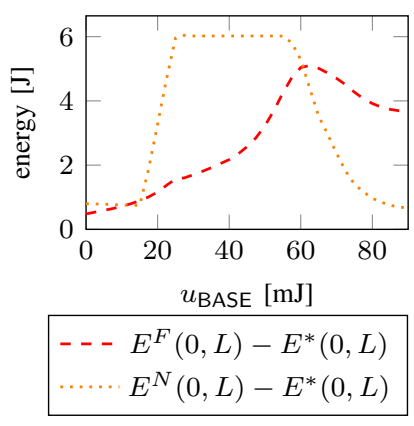

(a) Indoor Location L3 (b) Indoor Location L4

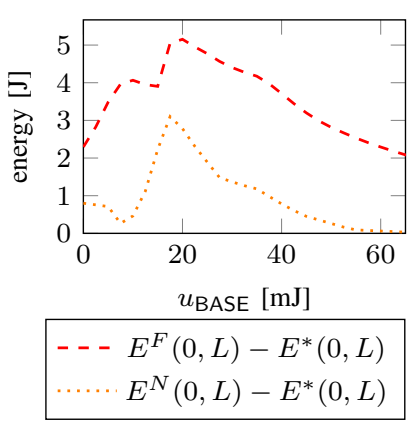

Fig. 4. Difference between the minimum of the total used backup energy $E^{*}$, and the practical solutions $E^{F}$ (model predictive control) and $E^{N}$ (baseline solution), for interval $[0, L)=[0,12 \cdot 144)$. Smaller is better

where we used the recommended target of stored energy $c=0.6$.

The experiments illustrate how the choice of base consumption impacts the backup battery usage at two locations, our energy management with a backup battery, and its reactivity to sudden and non-deterministic changes.

1) Experimental Setup: We used the system implementation as introduced in sec. VII, however a smaller rechargeable energy storage with capacity $B_{R}=1.35 \mathrm{~J}$ was assumed in order to adapt to the smaller amount of harvested energy. Two indoor locations were picked from the Indoor Solar Harvesting database [33], 'L3' and 'L4'. One day is used to calculate the harvested energy estimates, while the simulated time frame is 12 days, each with 144 10-minute intervals, i.e., $L=12 \cdot 144$. The predictive control horizon length is one hour, $T=6$. For both locations, we evaluate the impact the base consumption $u_{\text {BASE }}$ has on the total used backup energy $E(0, L)$. The base consumption $u_{\text {BASE }}$ is varied from $0 \mathrm{~J}$ to $90 \mathrm{~mJ}$ for location L3, and to $60 \mathrm{~mJ}$ for location $\mathrm{L} 4$.

Even though both indoor locations are located in the same room, the harvesting characteristics are fundamentally different. For the first location, L3, the harvester is placed on a table facing up, without many obstacles surrounding it. In this environment, the prediction of the future energy using EWMA is reasonably precise. For the second location, L4, the harvester is mounted on a wall facing west, and near a window. Close by, a curtain is sometimes partly obstructs the window. Here the EWMA predictor showed a large deviation between predicted and harvested energies, mainly because it could not predict the movement of the curtain.

2) Experimental Evaluation: Figure 4 visualizes the difference in total backup energy between the optimal clairvoyant solution $u^{*}(t)$, and the two implemented algorithms $u^{F}(t)$ (finite horizon control) and $u^{N}(t)$ (baseline).

For location L3, where the EWMA prediction is good, we see there is a prominent difference between the baseline and finite horizon solution, for low to medium values of the base consumption $u_{\mathrm{BASE}}$, namely less than $60 \mathrm{~mJ}$. When the $u_{\mathrm{BASE}}$ is larger than that value, the backup battery is used every time instance as $u^{*}(t)=u_{\mathrm{BASE}}$ for all $t \in[0, T)$. Thus, any solution which does not lead to wasted energy performs as good as 
the optimal one. The finite horizon solution is sub-optimal in this case, as the prediction algorithm and its pessimistic misspredictions lead to wasted energy.

At location L4, the finite horizon control solution underperforms the baseline. This is due to the almost useless predictions from the simple EWMA predictor. During the simulated days, there are both significant over- and underestimations of future energy. We therefore conclude that it does not make sense to use any prediction-based scheduler without a performant future energy predictor, and in these cases simple reactive power management solutions fit better.

In summary, the finite horizon control for energy harvesting systems with a backup battery performs well in terms of use of backup battery if the energy estimation is sufficiently precise, otherwise a simple reactive approach should be used.

\section{REAL WORLD EXPERIMENTS}

In this section, we further validate the realism of our nonideal model. To this end, we deployed the energy harvesting embedded system, introduced in sec. VII, in a controlled laboratory environment. We compare the simulated and measured harvested energy, consumed energy, and super-capacitor's charge. For comparison, we use the L2 norm of the difference between respective emulated and measured traces. We thus close the loop on the abstract model, considering non-ideal behavior, component characterization and measured system behavior under realistic conditions.

1) Experimental Setup: The Environment. Using the solar testbed [30], we closely recreate three days of indoor illumination conditions, as recorded at location 'L2' of the Indoor Solar Harvesting database [33] on 29 - 31 May 2018. The recreated trace features significant natural light, especially during morning hours. The solar test-bed enables this recreation, because it encapsulates the a photovoltaic panel and exposes it to desired illumination levels.

The three-day trace was slightly adjusted for practical purposes. First, the trace was sped up by a factor of 5 times, so the experiment lasted 14.4 hours. Then, the recorded trace was divided into two-minute intervals, over which the illumination level from the recorded trace was averaged, i.e., the illumination level was updated every two minutes. In order for the experiment to be more dynamic, such that we record the rechargeable energy storage getting fully charged and discharged, we scaled the illumination levels by a factor of 8 times. Functionally, this is equivalent to having an 8 times larger photovoltaic panel.

Practical System. The energy harvesting hardware and software as introduced in sec. VII-1 is used as the real world system. The 'power manager' updates the 'adaptive consumer' task every two minutes. The used consumption trace was determined by the finite horizon scheme with a non-perfect future energy estimate. The consumption varies from $2.01 \mathrm{~mW}$ $\left(2.01 \mathrm{~mW} \cdot 120 \mathrm{~s}=u_{\text {BASE }}\right)$ to $17.64 \mathrm{~mW}$. Current and voltage measurements are done using a RocketLogger [31], which measures in parallel: the harvested power, the rechargeable energy storage's voltage, and the system's power dissipation.

Simulated System. To compare our implemented hardwaresoftware system with model-based simulation results, we made

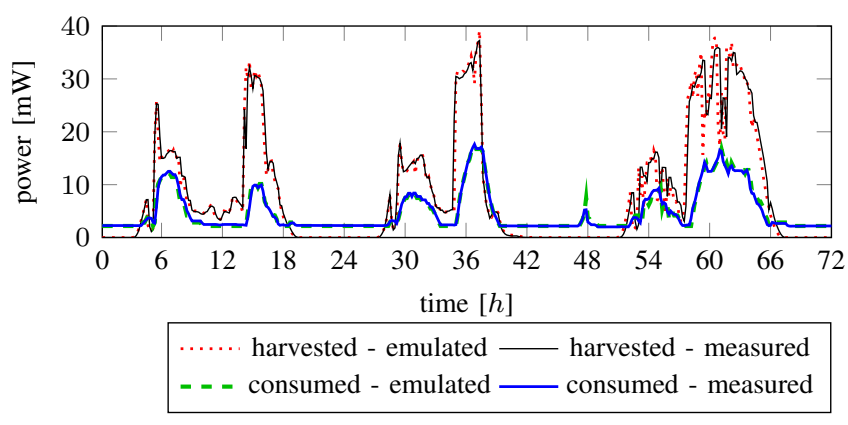

(a) Harvested and Consumed Power

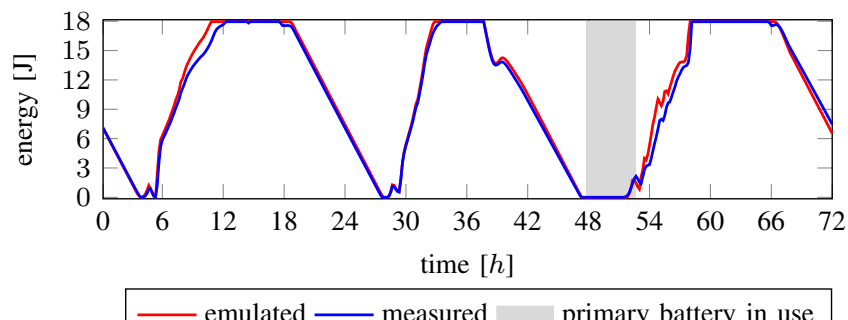

(b) Charge of the Rechargeable Energy Storage

Fig. 5. Comparison between the real-world embedded system and emulation results, over three days at indoor location L2.

use of the non-ideal model introduced in sec. VI, and considered the coefficients obtained by the component characterization as described in sec. VII-2: $\sigma_{p}=0.8529$ and $\sigma_{R}=0.9269$. We used the same trace of harvested energy and consumption, and emulated the rechargeable energy storage's charge.

2) Experimental Evaluation: Figure 5 shows the measured and simulated values side by side: the harvested and consumed power, and the charge of the super-capacitor. Energy values are obtained by multiplying the corresponding power with the 120 s time-interval length. The harvested energy's average L2 difference between measured and emulated results is $12.51 \mathrm{~mJ}$, which translates to a difference of $104.24 \mu \mathrm{W}$ in power. This difference is $1.16 \%$ of the average measured power. The L2 norm of the difference between the measured and emulated consumed energy is $2.75 \mathrm{~mJ}$ on average, corresponding to a $22.88 \mu \mathrm{W}$ difference in power. This constitutes a $0.47 \%$ difference to the average measured consumption.

Finally, the measured rechargeable energy storage is found to behave similarly to its emulated counterpart as well. The L2 difference between the two traces is $30.18 \mathrm{~mJ}$, which is $0.17 \%$ of the super-capacitor's maximal charge. Note that the backup battery was used once during this experiment, in the night between the second and third day. In summary, based on this comparison we can strongly corroborate the practical applicability of the proposed models and energy management algorithms. The differences between the abstract model and the considered non-ideal system behavior, and the actual implementation and measurements are negligible.

\section{Conclusion}

This paper is concerned with energy harvesting WSNs. In particular, we investigate energy management subsystems that make use of a backup battery in order to increase the efficiency 
and speed of a cold-start from an empty rechargeable energy storage, to retain state across restarts, or to allow uninterrupted operation for years or decades. To date, no energy control algorithm is known to maximize the lifetime of the node while guaranteeing a minimal energy use. Our proposed energy control algorithm optimizes a long-term utility function of the node under this constraint, if a perfect estimate of the future energy is available. Model predictive control is used to convert this optimal clairvoyant algorithm into a usable heuristic. Extensive trace-based simulation results as well as experiments with an implemented hardware solution are used to quantify and model non-ideal behavior, and to validate the usefulness of our approach in terms of guarantees, lifetime and run-time overhead.

Limitations of the approach are described as well, in particular if sufficiently precise energy estimates are not available. In such a case, a prediction-free algorithm may be more suitable. In a future work, we will investigate scenarios where energy estimations are not available, and quantify the associated loss in performance. In addition, we are looking into energy control algorithms based purely on statistical information on the energy harvesting source. Finally, we aim to illustrate our energy control scheme by realizing automatic control by means of an energy harvesting cyber-physical system.

\section{REFERENCES}

[1] F. K. Shaikh and S. Zeadally, "Energy harvesting in wireless sensor networks: A comprehensive review," Renewable and Sustainable Energy Reviews, vol. 55, pp. 1041-1054, 2016.

[2] B. Lucia, V. Balaji, A. Colin, K. Maeng, and E. Ruppel, "Intermittent computing: Challenges and opportunities," in 2nd Summit on Advances in Programming Languages (SNAPL 2017). Schloss Dagstuhl-LeibnizZentrum fuer Informatik, 2017.

[3] A. Gomez, L. Sigrist, M. Magno, L. Benini, and L. Thiele, "Dynamic energy burst scaling for transiently powered systems," in 2016 Design, Automation \& Test in Europe Conference \& Exhibition (DATE). IEEE, 2016, pp. 349-354.

[4] B. Ransford, J. Sorber, and K. Fu, "Mementos: System support for longrunning computation on rfid-scale devices," in Proceedings of the sixteenth international conference on Architectural support for programming languages and operating systems, 2011, pp. 159-170.

[5] D. Balsamo, A. S. Weddell, G. V. Merrett, B. M. Al-Hashimi, D. Brunelli, and L. Benini, "Hibernus: Sustaining computation during intermittent supply for energy-harvesting systems," IEEE Embedded Systems Letters, vol. 7, no. 1, pp. 15-18, 2014.

[6] N. Jackson, J. Adkins, and P. Dutta, "Capacity over capacitance for reliable energy harvesting sensors," in Proc. of the 18th International Conference on Information Processing in Sensor Networks, 2019, pp. 193-204.

[7] P. Visconti, R. Ferri, M. Pucciarelli, and E. Venere, "Development and characterization of a solar-based energy harvesting and power management system for a wsn node applied to optimized goods transport and storage," International Journal on Smart Sensing \& Intelligent Systems, vol. 9, no. 4, 2016.

[8] J. Jessen, M. Venzke, and V. Turau, "Design considerations for a universal smart energy module for energy harvesting in wireless sensor networks," in 2011 Proceedings of the Ninth International Workshop on Intelligent Solutions in Embedded Systems. IEEE, 2011, pp. 35-40.

[9] Texas Instruments, "Bq25505 ultra low-power boost charger with battery management and autonomous power multiplexer for primary battery in energy harvester applications," BQ25505 Datasheet, vol. 3, 2019.

[10] A. Kansal, J. Hsu, S. Zahedi, and M. B. Srivastava, "Power management in energy harvesting sensor networks," ACM Transactions on Embedded Computing Systems (TECS), vol. 6, no. 4, pp. 32-es, 2007.

[11] C. M. Vigorito, D. Ganesan, and A. G. Barto, "Adaptive control of duty cycling in energy-harvesting wireless sensor networks," in 2007 4th Annual IEEE Communications Society Conference on Sensor, Mesh and Ad Hoc Communications and Networks. IEEE, 2007, pp. 21-30.
[12] V. Sharma, U. Mukherji, V. Joseph, and S. Gupta, "Optimal energy management policies for energy harvesting sensor nodes," IEEE Transactions on Wireless Communications, vol. 9, no. 4, pp. 1326-1336, 2010.

[13] R. Ahmed, B. Buchli, S. Draskovic, L. Sigrist, P. Kumar, and L. Thiele, "Optimal power management with guaranteed minimum energy utilization for solar energy harvesting systems," ACM Transactions on Embedded Computing Systems (TECS), vol. 18, no. 4, pp. 1-26, 2019.

[14] C. Moser, L. Thiele, D. Brunelli, and L. Benini, "Adaptive power management for environmentally powered systems," IEEE Transactions on Computers, vol. 59, no. 4, pp. 478-491, 2009.

[15] T. Zhou and W. Sun, "Optimization of battery-supercapacitor hybrid energy storage station in wind/solar generation system," IEEE transactions on sustainable energy, vol. 5, no. 2, pp. 408-415, 2014.

[16] I. Atzeni, L. G. Ordóñez, G. Scutari, D. P. Palomar, and J. R. Fonollosa, "Demand-side management via distributed energy generation and storage optimization," IEEE Transactions on Smart Grid, vol. 4, no. 2, pp. 866876,2012

[17] L. Vračar, A. Prijić, D. Nešić, S. Dević, and Z. Prijić, "Photovoltaic energy harvesting wireless sensor node for telemetry applications optimized for low illumination levels," Electronics, vol. 5, no. 2, p. 26, 2016.

[18] B. Buchli, F. Sutton, J. Beutel, and L. Thiele, "Towards enabling uninterrupted long-term operation of solar energy harvesting embedded systems," in European Conference on Wireless Sensor Networks. Springer, 2014, pp. 66-83.

[19] _ "Dynamic power management for long-term energy neutral operation of solar energy harvesting systems," in Proceedings of the 12th ACM conference on embedded network sensor systems, 2014, pp. 31-45.

[20] P. Corke, P. Valencia, P. Sikka, T. Wark, and L. Overs, "Long-duration solar-powered wireless sensor networks," in Proceedings of the 4th workshop on Embedded networked sensors, 2007, pp. 33-37.

[21] J. Taneja, J. Jeong, and D. Culler, "Design, modeling, and capacity planning for micro-solar power sensor networks," in 2008 International Conference on Information Processing in Sensor Networks (ipsn 2008). IEEE, 2008, pp. 407-418.

[22] G. V. Merrett and B. M. Al-Hashimi, "Energy-driven computing: Rethinking the design of energy harvesting systems," in Design, Automation \& Test in Europe Conference \& Exhibition (DATE), 2017. IEEE, 2017, pp. $960-965$.

[23] S. Naderiparizi, A. N. Parks, Z. Kapetanovic, B. Ransford, and J. R. Smith, "Wispcam: A battery-free rfid camera," in 2015 IEEE International Conference on RFID (RFID). IEEE, 2015, pp. 166-173.

[24] S. DeBruin, B. Campbell, and P. Dutta, "Monjolo: An energy-harvesting energy meter architecture," in Proceedings of the 11th ACM Conference on Embedded Networked Sensor Systems, 2013, pp. 1-14.

[25] H. Jayakumar, A. Raha, and V. Raghunathan, "Quickrecall: A low overhead hw/sw approach for enabling computations across power cycles in transiently powered computers," in 201427 th International Conference on VLSI Design and 2014 13th International Conference on Embedded Systems. IEEE, 2014, pp. 330-335.

[26] D. Balsamo, A. Das, A. S. Weddell, D. Brunelli, B. M. Al-Hashimi, G. V. Merrett, and L. Benini, "Graceful performance modulation for powerneutral transient computing systems," IEEE Transactions on ComputerAided Design of Integrated Circuits and Systems, vol. 35, no. 5, pp. 738749,2016

[27] B. Buchli, P. Kumar, and L. Thiele, "Optimal power management with guaranteed minimum energy utilization for solar energy harvesting systems," in 2015 International Conference on Distributed Computing in Sensor Systems. IEEE, 2015, pp. 147-158.

[28] S. Chen, P. Sinha, N. B. Shroff, and C. Joo, "Finite-horizon energy allocation and routing scheme in rechargeable sensor networks," in 2011 Proceedings IEEE INFOCOM. IEEE, 2011, pp. 2273-2281.

[29] W. H. Kwon and S. H. Han, Receding horizon control: model predictive control for state models. Springer Science \& Business Media, 2006.

[30] L. Sigrist, "Design and instrumentation of environment-powered systems," Ph.D. dissertation, ETH Zurich, 2020.

[31] L. Sigrist, A. Gomez, R. Lim, S. Lippuner, M. Leubin, and L. Thiele, "Measurement and validation of energy harvesting iot devices," in Design, Automation \& Test in Europe Conference \& Exhibition (DATE), 2017. IEEE, 2017, pp. 1159-1164.

[32] D. R. Cox, "Prediction by exponentially weighted moving averages and related methods," Journal of the Royal Statistical Society: Series B (Methodological), vol. 23, no. 2, pp. 414-422, 1961.

[33] L. Sigrist, A. Gomez, and L. Thiele, "Dataset: Tracing indoor solar harvesting," in Proceedings of the 2nd Workshop on Data Acquisition To Analysis, 2019, pp. 47-50. 